\title{
Correspondence
}

\section{Urgent need for multi-site controlled trials for CMV pneumonia treatment in African children}

The paper by Jeena et al. on cytomegalovirus (CMV) in South African children with severe pneumonia, and the accompanying editorial by Gie and Goussard in the December 2017 issue of this Journal ${ }^{1,2}$ highlight a neglected but very important issue: ${ }^{3-6}$ Despite the availability of vaccines for Haemophilus influenzae type B (Hib) and Streptococcus pneumoniae and appropriate antibiotics, pneumonia remains the most important infectious cause of death in children globally. ${ }^{7}$ Our recent autopsy study from Zambia showed that while bacterial causes of pneumonia are important, there is a high burden of undiagnosed tuberculosis (TB), CMV and other infectious and non-infectious pathologies, including co-morbidities, resulting in fatal outcomes. ${ }^{5}$ World Health Organization guidelines focus on clinical management and antibiotic regimens that fail to address the key factors driving the continued high burden of pneumonia in children: 1) drug-resistant bacterial pneumonia, 2) undiagnosed TB or CMV pneumonia, 3) co-infection with multiple bacterial or viral pathogens or noncommunicable disease (NCD) co-morbidity, and 4) late referral and overall poor quality care due to lack of trained staff and equipment.

The first three factors can be addressed through improvements in diagnostics and-services. CMV diagnosis is complex, and Gie and Goussard rightly suggest that high fidelity diagnostics could be designed for CMV, exploiting polymerase chain reaction platforms such as those rolled out for TB or human immunodeficiency virus (HIV) viral loads to address this. ${ }^{2}$ Randomised controlled trials (RCTs) are needed to assess the impact of empirical treatment for some common pathogens in high-risk HIV-infected or -exposed infants, as has proved extremely successful with respect to cotrimoxazol prophylaxis and Pneumocystis jiroveci pneumonia. Could empirical treatment of CMV and TB, both ubiquitous highly immunomodulatory and immunologically dominant pathogens, and proven causes of paediatric pneumonia deaths, ${ }^{3,5}$ deliver broader benefits for African children?

The primary CMV drug, ganciclovir (GCV), causes neutropaenia, and its efficacy in treating CMV pneumonia in seriously ill African children has not been evaluated in RCTs. GCV is unavailable outside South Africa and is not the-standard of care across subSaharan Africa due to its high cost, and the lack of clinical case definitions for using the drug and indications as to when to stop treatment. Jeena et al.'s study adds to the growing body of evidence ${ }^{3-6}$ that presents a compelling case for conducting RCTs of GCV in African children to determine the efficacy, dose and treatment duration of GCV or its oral equivalent, valganciclovir, which is easily administered and hence lends itself to study. South African researchers with experience administering GCV to young infants should look to collaborate regionally to implement such trials, and funding bodies should recognise 1) that pneumonia is the most important infectious cause of death in children, and 2) that there are multiple causes that need novel diagnostics and therapeutic strategies.

John Tembo* Cinta Moraleda ${ }^{\ddagger}$ PABLo Rojo ${ }^{\ddagger}$ Alimudin ZumLA ${ }^{\mathbb{S}}$ MatTHEW BatestII

*Tongii Medical College, Huazhong Science and Technology University, Wuhan, China

tHerpeZ, University Teaching Hospital, Lusaka, Zambia, www.herpez.org

*Fundación para la Investigación Biomédica del Hospital Universitario 12 de Octubre, Madrid, Spain

$\mathbb{\$}$ Division of Infection and Immunity, University College London, and NIHR BRC at UCL Hospitals NHS Foundation Trust, London

II School of Life Sciences, University of Lincoln, Lincoln, UK e-mail:john.tembo@gmail.com http://dx.doi.org/10.5588/ijtld.17.0847

Conflicts of interest: none declared.

\section{References}

1 Jeena P M, Govender K, Parboosing R, Adhikari M. The significance of cytomegalovirus in children with pneumonia admitted for mechanical ventilation. Int J Tuberc Lung Dis 2017; 21: $1230-1236$

2 Gie R P, Goussard P. CMV pneumonia in HIV-infected and HIVuninfected infants: a neglected disease? Int J Tuberc Lung Dis 2017; 21: 1209-1210.

3 Chintu C, Mudenda V, Lucas S, et al. Lung diseases at necropsy in African children dying from respiratory illnesses: a descriptive necropsy study. Lancet 2002; 360: 985-990.

4 Goussard P, Kling S, Gie R P, et al. CMV pneumonia in HIVinfected ventilated infants. Pediatr Pulmonol 2010; 45: 650-655.

5 Bates M, Shibemba A, Mudenda V, et al. Burden of respiratory tract infections at post mortem in Zambian children. BMC Med 2016; 14: 99.

6 Tembo J, Kabwe M, Chilukutu L, et al. Prevalence and risk factors for betaherpesvirus DNAemia in children $>3$ weeks and $<2$ years of age admitted to a large referral hospital in subSaharan Africa. Clin Infect Dis 2015; 60: 423-431.

7 Liu L, Oza S, Hogan D, et al. Global, regional, and national causes of under-5 mortality in 2000-15: an updated systematic analysis with implications for the Sustainable Development Goals. Lancet 2016; 388: 3027-3035. 
Queries for jtld-22-04-22a_22b

This manuscript/text has been typeset from the submitted material. Please check this proof carefully to make sure there have been no font conversion errors or inadvertent formatting errors. Allen Press. 